Multiple Criteria Decision Making

Hatem Masri

Blanca Pérez-Gladish

Constantin Zopounidis Editors

Financial Decision

Aid Using Multiple

Criteria

Recent Models and Applications 


\title{
Distance Measures for Portfolio Selection
}

\author{
Joseph Andria, Giacomo di Tollo, and Arne Lokketangen
}

\begin{abstract}
The classical Markowitz approach to the portfolio selection problem (PSP) consists of selecting the portfolio that minimises the return variance for a given level of expected return. By solving the problem for different values of this expected return we obtain the Pareto efficient frontier, which is composed of nondominated portfolios. The final user has to discriminate amongst these points by resorting to an external criterion in order to decide which portfolio to invest in. We propose to define an external portfolio that corresponds to a desired criterion, and to assess its distance from the Markowitz frontier in market allowing for short-sellings or not. We show that this distance is able to give us useful information about out-ofsample performances. The pursued objective is to provide an operational method for discriminating amongst non-dominated portfolios considering the investors' preferences.
\end{abstract}

Keywords Decision aid - Distance measures - Financial models • Index tracking $\bullet$ Markowitz $\bullet$ Metaheuristics $\bullet$ Multiple criteria $\bullet$ Portfolio selection

Arne Lokketangen is deceased (10 June 2013).

J. Andria $(\bowtie)$

Dipartimento di Scienze Economiche, Aziendali e Statistiche, University of Palermo, Palermo, Italy

e-mail: joseph.andria@unipa.it

G. di Tollo

Dipartimento di Management, Universitá Ca’ Foscari, Venezia, Italy

e-mail: giacomo.ditollo@unive.it

A. Lokketangen

Molde University College, Molde, Norway

(C) Springer International Publishing AG 2018

H. Masri et al. (eds.), Financial Decision Aid Using Multiple Criteria, Multiple

Criteria Decision Making, https://doi.org/10.1007/978-3-319-68876-3_5 


\section{Introduction}

A common assumption in financial theory is that the information contained in historical prices can be used to assess the riskiness and expected return of assets: for a given asset $i$, its expected return is noted by $r_{i}$ and is given by the expected mean return $E\left(R_{i}\right)$; its risk is given by its return variance $\sigma_{i}^{2}$. Given an universe set $U$, a portfolio is described by a vector $X$, in which the $i$ th element represents the proportion of the total wealth invested in asset $i(i=1, \ldots, n)$. The covariance between assets $i$ and $j$ is denoted as $\sigma_{i j}=\sigma_{i} \sigma_{j} \rho_{i j}$ where $\rho_{i j}$ is the Pearson correlation coefficient; variance of portfolio $p$ is expressed as $\sigma_{p}^{2}=\sum_{i=1}^{n} \sum_{j=1}^{n} \sigma_{i j} x_{i} x_{j}$. The expected return is given by the weighted sum of asset returns $r_{p}=\sum_{i=1}^{n} r_{i} x_{i}$.

The basic formulation by Markowitz consists of minimising return variance by requesting that all capital has to be spent $\left(\sum x_{1}=1\right)$ and by imposing a lower bound on the required level of return $r_{e}\left(r_{p} \geq r_{e}\right.$ ) (Markowitz 1952). This formulation is solvable by standard Quadratic Programming solvers, and it is still referred to as the most used active approach to Portfolio Selection (Mansini et al. 2001). Operational constraints and preferences make this formulation more complex, resulting in a NPhard problem (Masini and Speranza 1999) for which standard exact approaches cannot be used to find the optimal solution. Several approaches can be used to find a satisfactory sub-optimal solution, and there exists a wide literature about solving the problem via approximated methods. Anyhow, the Markowitz approach provides us with a set of points, and decision makers have to choose amongst them by using an external criteria (di Tollo and Roli 2008).

Several approaches have been proposed to choose amongst the Pareto set: for example, a risk-free asset can be introduced, leading to an optimisation process that provides us the tangency portfolio (Black 1972). In another approach, it is possible to choose, out of the Efficient Frontier, the portfolio which maximise the reward-to-volatility ratio, also referred to as Sharpe Ratio (Sharpe 1970). In our work we want to discriminate amongst points over the Markowitz efficient frontier by using the distance between the Pareto front and a portfolio that represents the user's preferences. Several criteria could be used to define this portfolio, and in our work we are resorting to the Index Tracking portfolio: in this way we combine the two paradigms of active and passive portfolio management to define an ordinal multi-criteria preference system similar to the one proposed by Subbu et al. (2006), and we define a quantitative framework to understand which portfolios belonging to the Markowitz efficient frontier offer good performances out-of-sample. Our experimental analysis is carried as follows:

1. We define distance measures in order to compute the distance of an arbitrary portfolio from the Pareto frontier;

2. We determine a portfolio that meets the user preferences;

3. We find the Pareto portfolios belonging to the mean-variance frontier;

4. We compute distance of portfolio found at point (2) w.r.t. the frontier computed at point (3); 
5. We perform a correlation analysis between these measures and the portfolio's performance measures in order to decide which metric would be helpful to select a portfolio showing significant out-of-sample performances.

To this purpose we apply three distance measures (Lokketangen and Woodruff 2005):

- Manhattan Distance, given by the sum of asset weights differences between the two portfolios;

- Euclidean Distance, given by the squared sum of asset weights squared differences between the two portfolios;

- Hamming Distance, defined by introducing a binary variable to state if an asset is in the portfolio or not, and summing the absolute difference of these variables between the two portfolios;

As for the criterion used at point (2), in this work we assume that the user want to replicate the market behavior, hence minimising an error measure w.r.t. a financial index. The resulting portfolio is referred to as Index Tracking Portfolio and is widely used in the related literature (di Tollo and Maringer 2009). Please notice that our approach is robust and that other preferences and objective functions can be used and will be investigated in further works. Furthermore, please notice that we are not tackling a multi-objective Portfolio Selection that combines measures defined at points (2) and (3) as made by di Tollo et al. (2014), nor we want to provide an extensive comparison amongst solving algorithms.

In order to find portfolios at points (2) and (3), we use metaheuristics, which are general optimisation methods that can be used with arbitrary objective functions, that are easy to implement and that are able to find good sub-optimal solutions in reasonable amount of time. Furthermore, we are considering two different scenarios: the first is a market in which short-selling are allowed, and the latter in which there is a short-selling prohibition. Comparing the two cases is of practical relevance since short-sellings are not allowed in some countries and for some assets.

This chapter is organised as follows: the main approaches for portfolio selection will be outlined in Sect. 2, and the distance measures used in our approach will be outlined in Sect. 3. Metaheuristics will be introduced in Sect. 4 while Sect. 5 will detail the experimental phase. Section 6 concludes the chapter.

\section{Portfolio Strategies}

A common classification made by financial literature distinguish portfolio management strategies in active and passive strategies: an investor following an active strategy defines his/her own market expectations for the future; on the contrary, a passive management strategy aims to mimic the behavior of a given market index (or benchmark). The Markowitz model is the main specimen of active strategies; Index Tracking is an example of passive strategies. In this work we are combining 
those two approaches, and in what follows we are outlining the main features of both.

\subsection{The Markowitz Model and Its Extensions}

Let assume normal return distribution, infinitely divisible assets, no taxes, no transaction costs and short-selling prohibition: the Markowitz model can formulated as:

$$
\min \sum_{i=1}^{n} \sum_{j=1}^{n} \sigma_{i j} x_{i} x_{j}
$$

subject to

$$
\begin{aligned}
& \sum_{i=1}^{n}=r_{i} x_{i} \geq r_{e} \\
& \sum_{i=1}^{n} x_{i}=1 \\
& x_{i} \geq 0 \quad i=1, \ldots, n .
\end{aligned}
$$

By solving the problem for a set of values of $r_{e}$ it is possible to identify the non-dominated portfolios belonging to the efficient frontier, i.e., the Pareto-optimal portfolios that minimise risk for given levels of expected returns. Please notice that in this formulation an investor may only operate with long position, hence a basic extension of this model is possible by allowing negative $x_{i}$ values, i.e., by allowing short selling transactions. In this case the constraint (4) is replaced by the following:

$$
x_{i} \in \mathbb{R}
$$

and the resulting model is referred to as the Black model (Black 1972).

A further extension, proposed by Black himself, involves the introduction of an $n+1$ risk-free asset for which short selling operations are not allowed. Furthermore, in some countries additional constraints are imposed on short-selling: for instance in the U.S. the Regulation $T$ imposes margin requirements (collateral) to cover potential losses resulting from price movements (Jacobs et al. 2005). More in detail, assuming the collateral is a risk-free asset $n+1$, the proportion of money invested in it must be no less than a proportion $\gamma$ of the overall sum of the short positions, i.e.:

$$
x_{n+1}=-\gamma \cdot \sum_{i=1}^{n} \min \left\{0, x_{i}\right\} .
$$


Regulation T also imposes a limit in the total (short and long) exposure:

$$
\sum_{i=1}^{n}\left|x_{i}\right| \leq 2
$$

Moreover, the borrower may require a rebate of the interest earned on the proceeds gained from borrowing and selling stocks over the market (short-rebate h) (Jacobs et al. 2006). Taking into account the above mentioned aspects, the return of a long-short portfolio might be defined as

$$
r_{p}=\sum_{i=1}^{n}\left(r_{i}-h_{i} \cdot r_{c}\right) x_{i}
$$

with,

$$
h_{i}=0 \quad \text { if } \quad x>0,0 \leq h_{i} \leq 1 \quad \text { otherwise }
$$

where $c$ is the amount of money invested in cash-equivalent instruments. The resulting PSP can be expressed as follows:

$$
\begin{aligned}
& \min \sum_{i=1}^{n} \sum_{j=1}^{n} \sigma_{i j} x_{i} x_{j}, \\
& \sum_{i=1}^{n}\left(r_{i}-h_{i} \cdot r_{c}\right) x_{i} \geq r_{e} \\
& \sum_{i=1}^{n}\left|x_{i}\right|=1 \\
& -1 \leq x_{i} \leq 1 \\
& \sum_{i=1}^{n}\left|x_{i}\right| \leq 2 \\
& x_{n+1}=-\gamma \cdot \sum_{i=1}^{n} \min \left\{0, x_{i}\right\} \\
& h_{i}=0 \quad \text { if } \quad x>0,0 \leq h_{i} \leq 1 \quad \text { otherwise }
\end{aligned}
$$

Please notice that although several models have been proposed for dealing with short selling issues, the different regulatory practices hinder them to provide a unique modelling framework, hence imposing the need of general strategies that are robust to different objective functions and constraints. 


\subsection{The Index Tracking Model}

Differently from Markowitz, index tracking strategies aim to replicate the returns of a given market index over time without requiring a perfect match to the index (full replication): hence, they are a specimen of passive portfolio strategies. Index Tracking does not require any assumption about future asset returns, therefore it requires less implementation effort than an active portfolio strategy such as Markowitz. In its basic formulation, the index tracking problem consists in minimising over time the tracking error, defined as the return difference between the managed portfolio and the market index. As for the constraints, the same used in the Markowitz may still be used. Avoiding full replication makes the index less closely tracked, but resulting in a more efficient portfolio w.r.t. costs of construction, maintenance and management of the portfolio.

Let $r_{i, t}$ be the return of asset $i$ at time $t, r_{I, t}$ the return of index $I$ at time $t$ and $x_{i, t}$ the quantity of asset $i$ held at time $t$. As already stated, in the Index Tracking problem the objective is to find a portfolio that reproduces a pre-specified index $I$ over a given time horizon. Thus, given the return of portfolio $P, r_{P, t}=\sum_{i=1}^{n} r_{i, t} x_{i, t}$, this means that $r_{P, t}$ should be as close as possible to $r_{I, t}$ over a pre-specified period of time. This can be expressed by several objective functions. In the most common Tracking Error (TE) formulation the variance (VAR) of the difference between the tracking portfolio and the index return is used as a measure of deviation (Lobo et al. 2000; Roll 1992; Toy and Zurack 1989; Dahl et al. 1993; Franks 1992):

$$
\mathrm{TE}=\operatorname{VAR}\left(r_{p, t}-r_{I, t}\right)
$$

Other measures take into consideration the magnitude deviations from the index over a period of length $T$, such as:

$$
\mathrm{TE}=\frac{\left(\sum_{t=1}^{T}\left|r_{p, t}-r_{I, t}\right|^{\alpha}\right)^{1 / \alpha}}{T}
$$

or, as in Maringer and Oyewumi (2007)

$$
\mathrm{TE}=\left(\frac{\sum_{t=1}^{T}\left|r_{p, t}-r_{I, t}\right|^{\alpha}}{T}\right)^{1 / \alpha}
$$

Clearly, varying the value assigned to the parameter $\alpha$ can lead to different optimal results since tracking portfolios for a given $\alpha$ value could not be optimal also for another.

Nevertheless, in the TE problem one might want to penalize only negative deviations (downside) from the index while considering desirable the positive ones (upside). This point can be easily formalized if one seeks to maximize the portfolio 
return above the index return (excess return), i.e.:

$$
r_{\mathrm{excess}}=\sum_{t=1}^{T} \frac{r_{p, t}-r_{I, t}}{T}
$$

Actually, this measure has been mentioned, but not applied, by Beasley et al. (2003) and Gilli and Këllezi (2002).

There are basically two different approaches to overcome the issue of symmetrically penalize downside and upside deviations: one is to consider only those times $t$ over which the portfolio underperform the index, while the other consists in penalizing negative deviations in the objective function. The first approach has been proposed by Rudolf et al. (1999) where two risk measures are introduced, i.e., the Mean Absolute Deviation (which corresponds to Eq. (19) for $\alpha=1$ ) and a Min-Max criterion for which the maximum deviation between portfolio and index over the observation period is minimized.

In the second approach (Maringer 2008), a risk aversion coefficient is introduced following the more conventional purpose of maximizing the investor's utility objective function. If $\gamma$ represents the loss aversion parameter, the IT problem is formulated as follows:

$$
\begin{gathered}
\mathrm{TE}=\left(\frac{\sum_{t}\left(\Delta r_{t}\right)^{2}}{T}\right)^{1 / 2} \\
\Delta r_{t}= \begin{cases}r_{p}-r_{I} & \text { if } r_{p} \geq r_{I} \\
\left(r_{p}-r_{I}\right) \cdot \gamma & \text { if } r_{p}<r_{I}\end{cases}
\end{gathered}
$$

If both downside risk and upside excess return are merged into a single objective function, the optimization problem turns out to be:

$$
\min \lambda \mathrm{TE}-(1-\lambda) r_{\text {excess }} \quad 0 \leq \lambda \leq 1
$$

For a more detailed review of the topic we refer the reader to di Tollo and Maringer (2009).

\section{Distance Measures: Markowitz and Index Tracking}

The main idea behind this work is to study the distance between Mean Variance portfolios and Index Tracking ones, leading to a multi-criteria formulation that combines active and passive strategies. Some attempts to combine these two paradigms have been proposed in portfolio literature: for example multi-objective optimization problems with and index tracking approach has been introduced by 
Steuer et al. (2005) and solved along with Sharpe Ratio maximisation (Roll 1992) as well as with downside risk (Yu et al. 2006).

Other multi-objective approaches have been suggested by Jorion (2003), and di Tollo et al. (2014) define a multi-objective framework in which the three measures to be optimised are risk, variance and tracking error. All these approaches employ a multi-objective formulation of the problem. Our idea instead is first to solve separately the different optimisation problems coming from the two approaches, and then combining them in a multi-criteria approach able to express the user's preferences: first we determine the index tracking portfolio; then, we compute the efficient mean-variance frontier. Afterwards, for each point belonging to the mean-variance frontier we compute three distance measures w.r.t. the index tracking portfolio, and eventually we study the correlation between those measures and the out-of-sample performances (i.e., we are not using the aforementioned distance measures as objectives).

Let the Mean Variance Portfolio be a vector $M V=\left(m v_{1} \ldots m v_{n}\right)$, where $m v_{i}$ is the fraction invested in the $i$ th asset. Let the Index Tracking Portfolio be the vector $I T=\left(i t_{1} \ldots i t_{n}\right)$. We define the following distance measures:

- Manhattan Distance $M D=\sum_{i}^{n}\left|M V_{i}-I T_{i}\right|$;

- Euclidean Distance ED $\left.=\sqrt{(} \sum_{i}^{n}\left(M V_{i}-I T_{i}\right)^{2}\right)$;

- Hamming Distance HD $=\left|\sum_{i}^{n}\left(z i t_{i}-z m v_{i}\right)\right|$, where $z i t_{i}=\operatorname{ceil}\left(i t_{i}\right)$ and $z m v_{i}=$ $\operatorname{ceil}\left(m v_{i}\right)$;

defined in order to determine if a solution belongs or not to an admissible set of an investors' portfolio preferences (Lokketangen and Woodruff 2005). We remark that, in terms of Mean Variance analysis, the Index Tracking portfolio is always inefficient, since it has highest variance than the point on the Mean Variance with the same return. Nevertheless, it has good formal properties, and there is evidence that it performs well in terms of out-of-sample analysis. Also Mean Variance portfolios perform well out-of-sample, hence an approach that combines both approaches is desirable.

\section{Metaheuristics}

When solving an optimisation problem we may either use an exact or an approximated approach, the choice depending on the complexity and on the dimension of the problem, and on the desired target that one wants to achieve. Exact methods generally adopt a deterministic approach which guarantee the user to find the optimum of a problem, with proof of optimality. Examples of these approaches are: linear programming (Milano and Trick 2004), mixed integer-linear programming (Benati and Rizzi 2007), dynamic programming (Li and Ng 2000) and others.

Unfortunately, conventional optimization algorithms are not efficient when we attempt to cope with complex real world problems, which are generally NP-Hard and whose exact solution would require an unbearable computational time. In these 
cases, one has to resort to heuristic procedures which produce a good solutions in reasonable amount of computational time, but without proofing the optimal quality of their solution.

A generalisation of heuristics is the concept of metaheuristics (Blum and Roli 2003), that embed basic heuristic methods in a higher level framework in order to efficiently and effectively exploring a search space. They can be defined as highlevel strategies that coordinate the action of subordinated heuristics in order to find solution(s) for the problem. They are not problem-specific, hence they can be used in a variety of problems and formulations, such as, e.g., the problem at hand, in which we have used a metaheuristic approach, namely Threshold Acceptance (Gilli and Këllezi 2002) to find the Mean Variance efficient frontier and the Index Tracking Portfolio.

Threshold accepting is a metaheuristic in which degrading moves can be accepted if the cost difference between current and new solution is within a given threshold, whose value is decreased to zero over the different epochs. In order to apply this metaheuristic to the problem at hand, we have defined its local-search components as follows:

- Search Space The search space is composed by all portfolios whose asset values belong to the closed interval $[0,1]$ and sum to one. We do not consider other constraints since our objective is to study the interaction of the diverse objective functions rather than to test how different constraints can have an impact on the optimization process. Anyhow, our approach is robust to take into account further constraints.

- Neighborhood relations A fraction (step) of the asset is transferred from asset $a$ to asset $b$. If asset $b$ is not hold, then it is included in the portfolio. When an asset exhibits negative sign, then a zero value is given and other values are normalised accordingly. If there is an attempt to decrease the share of an asset being set to zero, the asset is deleted and other assets are normalised accordingly. The asset whose share is to be increased must be chosen so that $x_{i}+$ step $\leq 1$; if there is no asset that satisfy this constraint, step value is modified accordingly.

- Initial solution The starting solution is created randomly in order to satisfy all constraints in the formulation;

- Cost Function For Markowitz portfolios we use a penalty approach (Corazza et al. 2012) in which the cost function is given by the sum of the portfolio variance (risk) and the degree of violation of the return constraint; for Index Tracking portfolios we do not add any penalty to the objective function, which is the tracking error defined as (19);

- Local Search Strategies Threshold Accepting algorithm was implemented with the following settings: Iterations $=10,000$, Restart $=20$, Epochs $=5$. These parameters have been estimated by F-Race (Birattari et al. 2010). 


\section{Experimental Analysis}

In this section, we introduce a methodology for finding (or testing) mean-variance efficient points that are close enough to other pre-specified investment criteria and constraints. In particular, after having determined Markowitz mean-variance portfolios, we determine the portfolio that meets the investor requirement best, we compute the distance of this portfolio from mean-variance ones, and we use this information to select the portfolio to invest in. This approach leads to a multi-criteria decision-making problem.

As for the portfolio that meets the investor requirements, we assume that the investor wants to follow as close as possible the market, hence we use the Index Tracking Portfolio. We have used metaheuristics to find both mean-variance portfolios and index tracking portfolio. Please notice that metaheuristics may be used in order to find portfolio which are optimal w.r.t. any other user's requirements. Further works will be devoted to use other objective functions and preferences.

After having determined the points, we compute distances between each Markowitz portfolio and the Index Tracking portfolios. To this aim we have used the three distance measures defined in Sect. 3. Eventually we investigate the out-ofsample correlation between the computed distance measures and the mean-variance portfolio performances.

Threshold accepting has been implemented in Matlab. As for the Mean-Variance Portfolios, TA has been able to find portfolios that are comparable to the global optimum found by MOSEK (http://docs.mosek.com/7.0/toolbox/) for all instances taken into account, but with lower computational time.

We have performed our experiments on three benchmarks from the repository ORlib, ${ }^{1}$ that includes 290 weekly quotations of the following market indexes: Hang Seng (containing 31 assets), Dax100 (containing 85 assets), and FTSE100 (containing 89 assets). For each instance the Unconstrained Efficient Frontier (UEF) has been derived using 50 equally distanced values for the expected return $r_{e}$. Prices contained in the four benchmarks have been converted into return by using the logarithmic formula: $r_{i, t}=\ln \frac{S_{i, t+1}}{S_{i, t}}$, where $r_{i, t}$ is the return of asset $i$ at time $t$ and $S_{i, t}$ is the price of asset $i$ at time $t$. Furthermore, as in Gilli et al. (2011), we have performed comparisons between in-sample and out-of-sample analysis over subsets (time windows) of the weekly observations: we have defined seven starting points $t_{i}$, corresponding to the $(1+20 \cdot i)$ th weekly observation, $(i=0 \ldots 6)$. For each starting point we have used observations $t_{i}$ through $t_{i}+149$ as in-sample data, and $t_{i}+150$ through $t_{i}+170$ as out-of-sample data.

Our first investigation involves a correlation analysis between portfolio return and tracking error. In detail, a correlation study has been performed on the following variables:

(a) in sample Tracking Error;

(b) out of sample Tracking Error;

\footnotetext{
${ }^{1}$ http://mscmga.ms.ai.ac.uk/orlib/Jeb/portfolio.html.
} 
(c) in sample Return;

(d) out of sample Return.

By analysing results on all instances and on all temporal windows, we have remarked that Tracking Error (both in sample and out of sample) and in sample Return are positively correlated and in the Long-Short case the correlation is higher than the Long-Only case (see Table 1). This clearly indicates a case in which the two criteria are clearly conflicting to each other. But, more interestingly, we have also found small correlations between out of sample Return and the three other measures, especially on the Long Only case, indicating that none of the three measure may be considered as a valid proxy to forecast future returns. Hence, an analysis of the distance measures may be helpful to understand whether we could use them as proxy.

In what follows $\rho$ represents the Pearson correlation between the two metrics between parenthesis; HD represents the Hamming distance; MD represents the Manhattan distance; ED represents the Euclidean Distance. $\sigma$ represents the portfolio return variance; TE represents the Tracking Error and $r_{P}$ the return of portfolio. The three performance measures are reported w.r.t. in sample (IS) and out of sample (OS) data.

Then we have carried out a correlation analysis between each of the computed distance measures and the out-of-sample performance of mean-variance portfolios: out-of-sample return, out-of-sample variance and out-of-sample tracking error. Correlation amongst these measures are reported in Table 2, in which we report the mean and standard deviation of Pearson correlation values over all time windows taken into account (i.e., we computed a correlation coefficient for each time window).

A first quick conclusion of the above results is that the Hamming distance is often negatively correlated with all the other metrics in two instances out of three in the Long-Short case. When considering the Long-Only case the correlation is better positively defined, although frequently close to zero thus too instable to be used as a predictor of futrure returns.

More in detail, the metric which appear to be more correlated to return and variance is the Manhattan distance (in the Long-Short case this correlation is higher than the Long Only case). Then this would imply that the bigger this distance, the more the performance of the index-tracked portfolio is likely to be good in terms of mean-variance. Furthermore, it is worth noting that the majority of the distance metrics are strongly correlated to each other with the only exception being, as said above, the Hamming distance. This means that introducing all the other measures as complementary criteria into the portfolio selection problem does not provide any additional insight.

The aim of analysing the correlation amongst distance measures and performance measures is twofold. At first we want to understand whether the diverse measures lead to the same information (or not): highly correlated distance measures would imply that the information contained in the diverse definitions is similar (or different). Then, we want to use the information contained in the diverse distance 


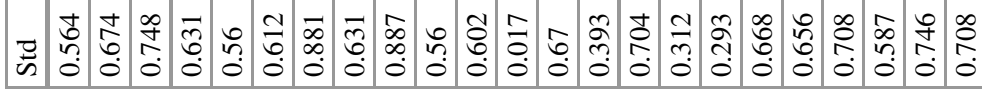

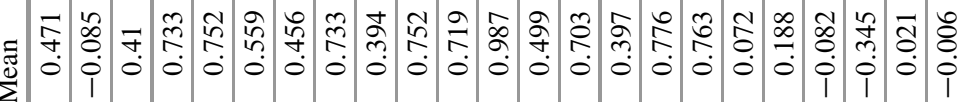 $3 \sum^{2}$}

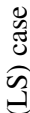

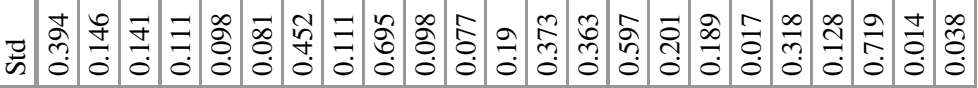

言

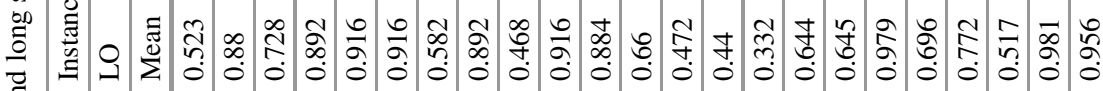

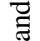

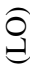

함

흫

言

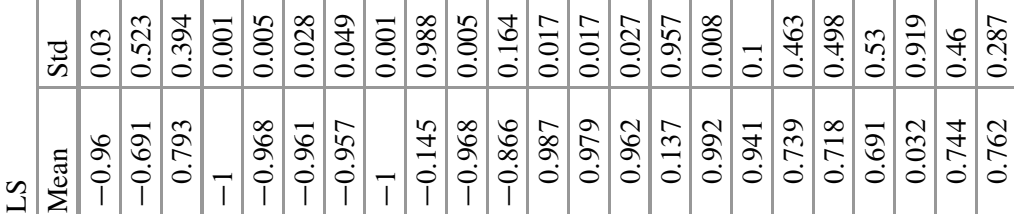

究

巳્ટ

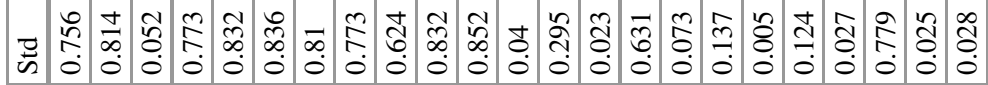

꾜

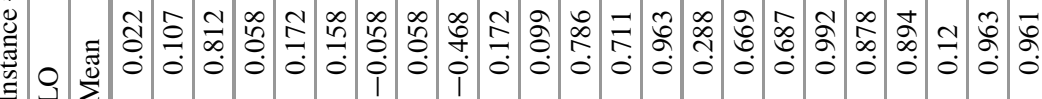

这

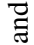

s

灵

छ

迟

ง

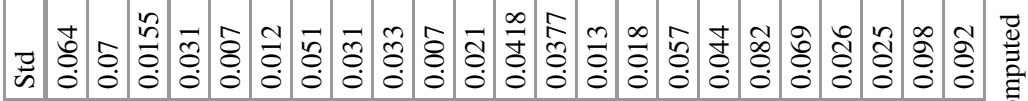

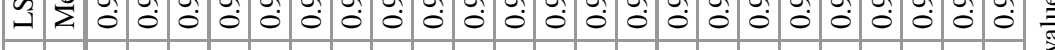

इ

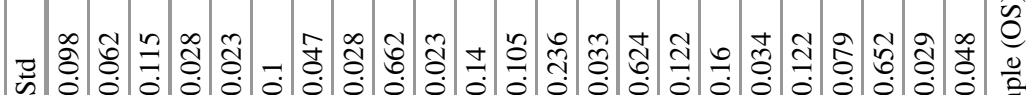

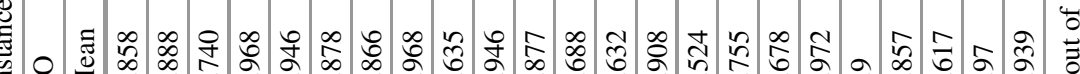

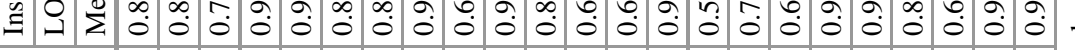

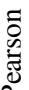

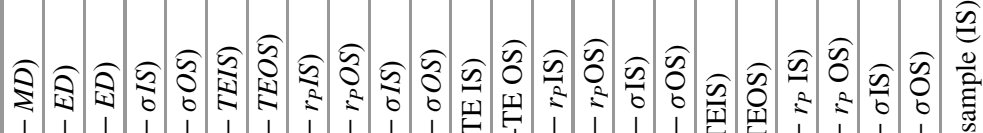

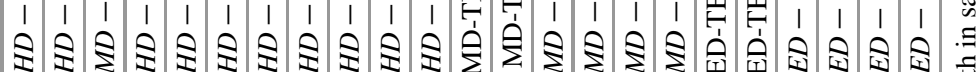

高 


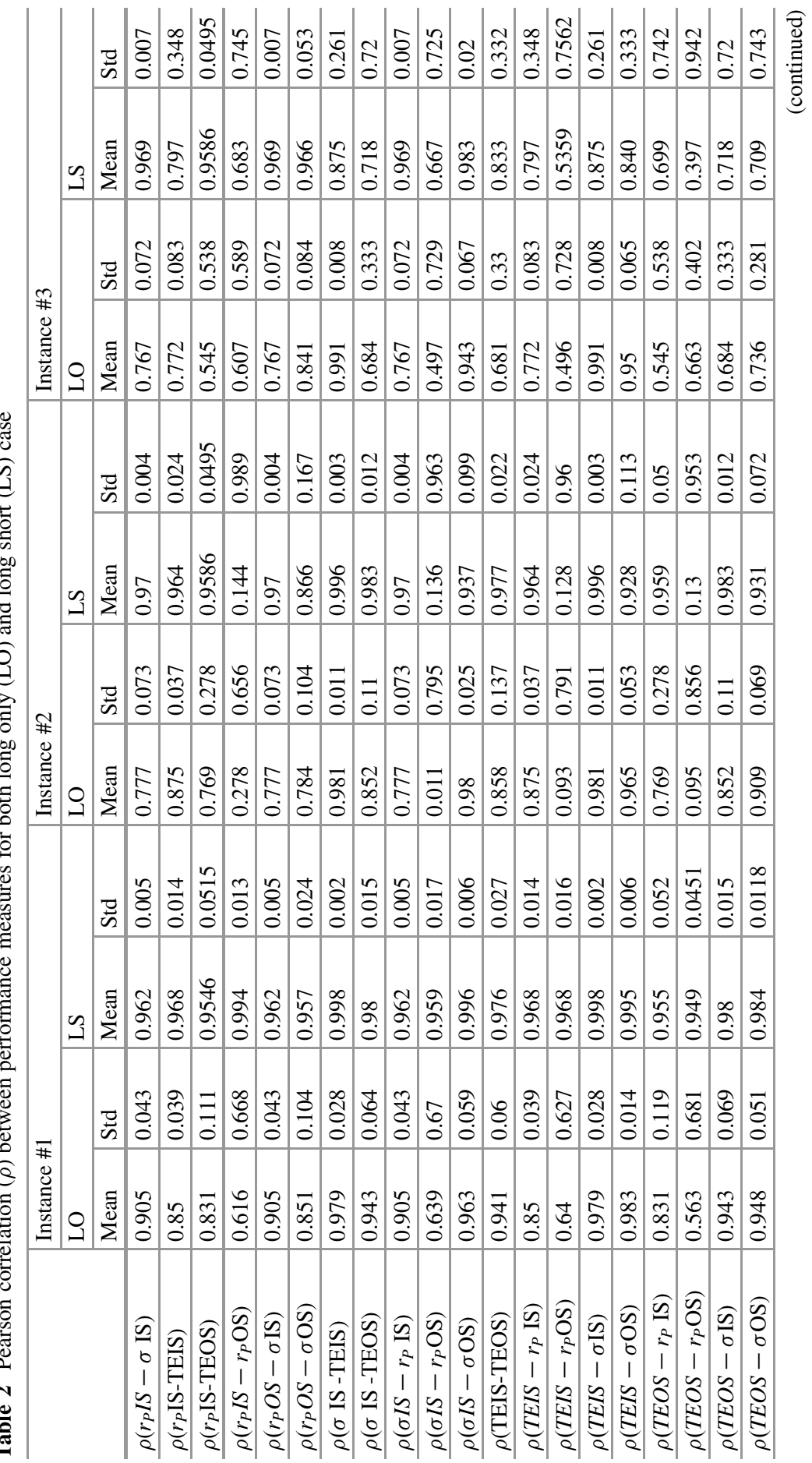




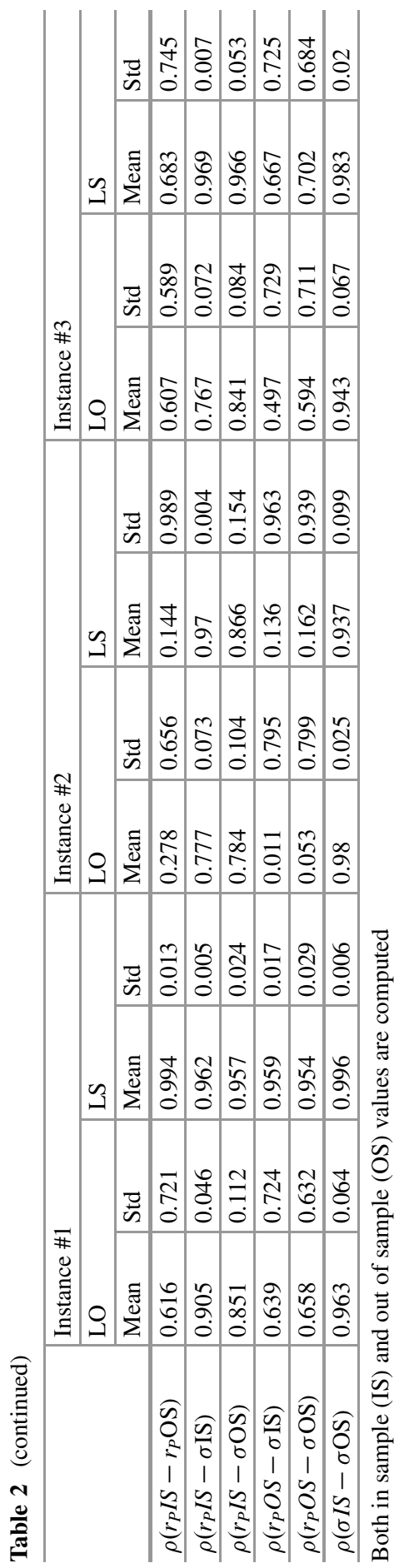


measures as operational suggestion about the investment to be performed, w.r.t. the criteria chosen by the investor. In a nutshell, if we state that a distance measure is highly correlated with out-of-sample return, and the investor is willing to maximise the return of its portfolio, he has to choose a portfolio with big distance measure. The same happen with other performance measures. The key point is to understand which distance measure to use.

At first, we remark that the three distance measures are highly correlated when considering the smallest instance; less when considering the bigger ones. The magnitude of this correlation is higher on the Long Short case. Hence, we cannot state a-priori that considering every distance measure lead to the same operational strategy. We should consider the correlation of every distance measure introduced with our performance measures: portfolio return, return variance and tracking error. We consider both in-sample and out-of-sample correlations, but in order to avoid overfitting we are more interested in out-of-sample performances, with a focus on future return.

If we consider returns, in the Long-Short case the Euclidean distance is highly correlated with both in-sample and out-of-sample returns on the smallest instance; this does not happen with respect to the other two instances, where the correlation is unstable, and the standard variation anyhow too big. In the Long-Only case instead the correlation is always positive, even though the magnitude is not high. In this case the Manhattan distance is the best metric, and when using Hamming distance we cannot even determine a unique sign.

As for variance, Manhattan distance is the best correlated over the three instances in-sample. This also held for out-of-sample analysis, in which, in two Long-Short cases out of three, the standard deviation is even smaller than the in-sample case. We remark that, the correlation between all distance measures taken into account and the portfolio return variance is high, with low standard variation for the smallest instance. The same does not happen when considering bigger-sized instances, where nothing can be said about the sign of the correlation, and whose standard deviations are higher over the considered time-windows. Also in this case, the Manhattan distance lead us to the highest and most stable correlation, and for the Long Only case we remark also that the Manhattan distance is the most stable, though with a smaller magnitude than the Long Short case.

We may conclude that the Manhattan distance between the Mean-Variance portfolios and the Index tracking one is the most capable to give the user insights about operational strategies: there exist a good correlation between Manhattan Distance and out-of-sample return, and this phenomenon is more significant when considering Short-Selling. Hence, we can state that this analysis leads us to choose, out of the Markowitz frontier of a market allowing short selling, the portfolio with a high Manhattan distance to the Index Tracking portfolio. 


\section{Conclusion}

In this work we have examined the out-of sample performances of mean-variance portfolios by analysing their distance to a non-dominated point that may represent the preference of an investor. To this aim, we have chosen as non-dominated point the Index-Tracking portfolio, which attempts to replicate an index' behavior: in this way we have combined active and passive portfolio management strategies.

We have remarked that the analysis of distance measures may lead to good operational advices when considering a market that allows short selling, and that different distance measures may lead to different analysis. Hence, we have assessed that the Manhattan strategy is the one that leads to more significant operational advices.

As further research we want to select other portfolios to express an investor's preference. Our analysis will be devoted to $\mathrm{VaR}$ and equally weighted portfolios, which are important for regulatory and operational issues. Furthermore, we will apply non additive measures, such as the Choquet integral, to investigate how the different criteria interact to each other.

Furthermore, we will extend our analysis to different instances and formulations. This will trigger the need of devising diverse solving approaches, thus giving rise to the necessity of performing an experimental comparison amongst different methods and algorithms.

\section{References}

Beasley, J. E., Meade, N., \& Chang, T. J. (2003). An evolutionary heuristic for the index tracking problem. European Journal of Operational Research, 148(3), 621-643.

Benati, S., \& Rizzi, R. (2007). A mixed integer linear programming formulation of the optimal mean/value-at-risk portfolio problem. European Journal of Operational Research, 176(1), 423-434.

Birattari, M., Yuan, Y., Balaprakash, P., \& Stützle, T. (2010). F-Race and iterated F-Race: An overview. In Experimental methods for the analysis of optimization algorithms (pp. 311-336). Berlin: Springer.

Black, F. (1972). Capital market equilibrium with restricted borrowing. Journal of Business, 45(3), 444-455.

Blum, C., \& Roli, A. (2003). Metaheuristics in combinatorial optimization: Overview and conceptual comparison. ACM Computing Surveys, 35(3), 268-308.

Corazza, M., Fasano, G., \& Gusso, R. (2012). Portfolio selection with an alternative measure of risk: Computational performances of particle swarm optimization and genetic algorithms. In Mathematical and statistical methods for actuarial sciences and finance (pp. 123-130). Milan: Springer.

Dahl, H., Meeraus, A., \& Zenios, S. A. (1993). Some financial optimization models: I risk management. In Financial optimization (pp. 3-36). Cambridge: Cambridge University Press.

di Tollo, G., \& Maringer, D. (2009). Metaheuristics for the index tracking problem. In Metaheuristics in the service industry (pp. 127-154). Berlin: Springer.

di Tollo, G., \& Roli, A. (2008). Metaheuristics for the portfolio selection problem. International Journal of Operations Research, 5(1), 443-458. 
di Tollo, G., Stützle, T., \& Birattari, M. (2014). A metaheuristic multi-criteria optimisation approach to portfolio selection. Journal of Applied Operational Research, 6(4), 222-242.

Franks, E. C. (1992). Targeting excess-of-benchmark returns. Journal of Portfolio Management, $18(4), 6-12$.

Gilli, M., \& Këllezi, K. (2002). The threshold accepting heuristic for index tracking. In Financial engineering, e-commerce and supply chain (pp. 1-18). New York: Springer.

Gilli, M., Schumann, E., di Tollo, G., \& Cabej, G. (2011). Constructing 130/30-portfolios with the omega ratio. Journal of Asset Management, 12(2), 94-108.

Jacobs, B., Levy, K. N., \& Markowitz, H. (2005). Portfolio optimization with factors, scenarios and realistic short positions. Operations Research, 53(4), 586-599.

Jacobs, B., Levy, K. N., \& Markowitz, H. (2006). Trimability and fast optimization of long-short portfolios. Financial Analysts Journal, 62(2), 36-46.

Jorion, P. (2003). Portfolio optimization with tracking-error constraints. Financial Analysts Journal, 59(5), 70-82.

Li, D., \& Ng, W. L. (2000). Optimal dynamic portfolio selection: Multiperiod mean-variance formulation. Mathematical Finance, 10(3), 387-406.

Lobo, M. S., Fazel, M., \& Boyd, S. (2000). Portfolio optimization with linear and fixed transaction costs and bounds on risk. Mimeo. Information Systems Laboratory, Stanford University.

Lokketangen, A., \& Woodruff, D. L. (2005). A distance function to support optimized selection decisions. Decision Support Systems, 39(3), 345-354.

Mansini, R., Ogryczak, W., \& Speranza, M. G. (2003). LP solvable models for portfolio optimization: A classification and computational comparison. IMA Journal of Management Mathematics, 14(3), 187-220.

Mansini, R., \& Speranza, M. G. (1999). Heuristic algorithms for the portfolio selection problem with minimum transaction lots. European Journal of Operational Research, 114, 219-233.

Maringer, D. (2008). Constrained index tracking under loss aversion using differential evolution. In Natural computing in computational finance (pp. 7-24). Berlin: Springer.

Maringer, D., \& Oyewumi, O. (2007). Index tracking with constrained portfolios. Intelligent Systems in Accounting, Finance \& Management, 15(1-2), 57-71.

Markowitz, H. (1952). Portfolio selection. Journal of Finance, 7(1), 77-91.

Milano, M., \& Trick, M. (2004). Constraint and integer programming (pp. 1-31). New York: Springer

Roll, R. (1992). A mean/variance analysis of tracking error. The Journal of Portfolio Management, $18(4), 13-22$.

Rudolf, M., Wolter, H. J., \& Zimmermann, H. (1999). A linear model for tracking error minimization. Journal of Banking \& Finance, 23(1), 85-103.

Sharpe, W. F. (1970). Portfolio theory and capital markets. New York: McGraw Hill.

Steuer, R. E., Qi, Y., \& Hirschberger, M. (2005). Multiple objectives in portfolio selection. Journal of Financial Decision Making, 1(1), 5-20.

Subbu, R., Bonissone, P. P., Eklund, N., Bollapragadaa, S., \& Chalermkraivuth, K. (2005). Multiobjective financial portfolio design: A hybrid evolutionary approach. In Proceedings of the 2005 IEEE Congress on Evolutionary Computation (pp. 1722-1729)

Toy, W. W., \& Zurack, M. A. (1989). Tracking the Euro-Pac index Journal of Portfolio Management, 15(2), 55-58.

Yu, L., Zhang, S., \& Zhou, X. (2006). A downside risk analysis based on financial index tracking models. In Stochastic finance (pp. 213-236). Boston, MA: Springer. 